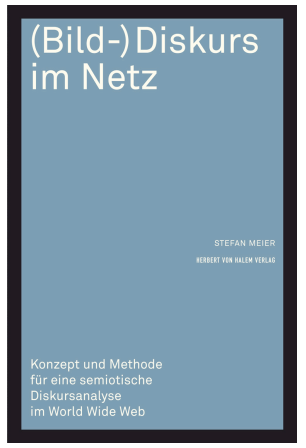

\begin{tabular}{l}
\hline Stefan Meier \\
(Bild-)Diskurs im Netz \\
Konzept und Methode für eine semiotische Diskursanalyse im World Wide Web \\
\hline Köln: Herbert von Halem, 2008. 480 Seiten \\
ISBN: 978-3-938258-83-5 \\
€ 32.-; CHF 53.40
\end{tabular}

\title{
Über die (visuelle) Konstitution von Onlinediskursen und ihre empirische Untersuchung
}

So alläglich und selbstverständlich Onlinekommunikation für viele Menschen ist - der empirischen Kommunikations- und Medienforschung fällt es weiterhin schwer, der Komplexität webbasierter Kommunikationsprozesse gerecht zu werden und geeignete Methoden und Instrumente für ihre Analyse zu entwickeln. Ein viel beschrittener (Aus-)Weg besteht darin, spezifische mediale Phänomene und Angebote zu fokussieren und ihre Merkmale und Strukturen bzw. ihre Nutzung oder Aneignung in den Blick zu nehmen. Der Chemnitzer Medienwissenschaftler Stefan Meier verspricht hingegen ein "holistisches» Vorgehen und weckt damit vermutlich das Interesse all jener, die in den so beleuchteten Teilausschnitten ein nur unvollkommenes Bild dessen konturiert sehen, was Onlinekommunikation im Besonderen ausmacht: Hypertext, Intertextualität und Multimedialität.

Der Autor nähert sich der Onlinekommunikation aus diskurstheoretischer Perspektive, integriert jedoch ebenso Ansätze der (Sozio-)Semiotik und der Wissenssoziologie. Ziel ist es, einen diskurstheoretischen Zugriff auf Onlinekommunikation zu erarbeiten, der ihren Bedingungen und vielfältigen Ausdrucksweisen gerecht wird. Ein Schwerpunkt liegt dementsprechend in der Integration visueller Zeichen und Kommunikationsformen. Darüber hinaus kann als forschungsleitende Motivation die Frage gelten, inwiefern den Einzelkommunikaten diskursbedingte Gestaltungsprinzipien unterliegen. Die Dissertation von Stefan Meier ist somit als Versuch zu lesen, Konzept und Methode für eine Forschungsperspektive zu entwickeln, die thematisch (d.h. an Diskursen) ansetzt und auf dieser Basis übergreifende Musterhaftigkeiten 
im «online-kommunikativen Zeichenhandeln» der Akteure/-innen zu bestimmen vermag. Von zentraler Bedeutung ist für Meier hierbei das soziosemiotische Konzept der «Multimodalität», welches «(...) Texte als eine Kombination unterschiedlicher Zeichenmodalitäten (Bild, Sprache, Ton) [fasst, W.R.], die gemäß ihren semiotischen Ressourcen (z.B. Farbgebung/Beleuchtung, Wortbildung/Semantik, Atmosphäre/Musik) als ganzheitliche Kommunikate zum Einsatz kommen und als solche auch in der Analyse zu behandeln sind» (158). Der Autor erprobt und illustriert seinen Ansatz einer semiotischen Diskursanalyse am Beispiel des Online-Diskurses zur zweiten Version der so genannten "Wehrmachtsausstellung» des Hamburger Instituts für Sozialforschung, die von 2002 bis 2004 zu sehen war und in der Öffentlichkeit starke Beachtung fand.

Die Arbeit gliedert sich in zwei Teile. Der erste Teil «Konzeptualisierungen» hebt mit einer Einführung in die sozialphilosophisch geprägte Diskurstheorie von Foucault an. Seiner methodisch-methodologischen Orientierung folgend, stellt der Autor im Anschluss Konzepte der angewandten Diskursforschung vor, die er zunächst separat bespricht. Ihre Synthese finden diese Ausführungen im Abschnitt über transdisziplinäre Fragestellungen an (Online)Diskurse. Hierbei setzt Stefan Meier drei Schwerpunkte, die sich zum einen auf die diskursive Konstituierung von (kollektivem) Wissen, zum anderen auf die Akteur/innen und ihre Interaktionsaktionsrollen sowie inre Imagearbeit und zum dritten auf die Intertextualität der Kommunikate richten. Hervorzuheben sind die sich anschließenden Ausführungen zum Zeichenhandeln als multimodale Diskurspraxis. Auch unabhängig von der fokussierten Diskursperspektive geben diese Einblick in die Bezüge und das Zusammenspiel zwischen Bild, Text und Webdesign als Mittel des online-kommunikativen Handelns. So schlägt Meier in Anlehnung an Stöckl beispielsweise zehn «Richtlinien zur Bestimmung von Bildfunktionen im Netz» (140ff.) vor.

Im zweiten Teil «Methodologie, Methode und Analysen» entfaltet der Autor sein semiotisches Konzept für die Untersuchung sozialer Diskurse. Als Verbindungsglied zwischen individueller und interpersonaler Kommunikation (Mikroebene) und gesellschaftlichem Diskurs (Makroebene) dient inm unter Rekurs auf soziosemiotische sowie kodetheoretische Argumentationen ein «multimodaler Musterbegriff». Demnach erlangen Zeichen «(...) ihre überindividuellen Bedeutungen durch kollektive Zuschreibungen, die nach Eco über konventionelle und kulturelle Regelsysteme (Kodes) vermittelt sind (...)» (211). Die Analyse der Diskursmuster soll nach Meier in einem herme- 
neutisch-interpretativen Verfahren und durch den Vergleich der multimodalen Texte auf fünf Ebenen erfolgen. Unterschieden werden Argumentationsbzw. sprachliche und bildliche Signifikationsmuster; Deutungs- und Interpretationsmuster sowie Kollektivsymboliken; intertextuelle Verknüpfungsmuster; Stilmuster sowie Rollen- und Verhaltensmuster. Ihre Identifikation setzt die Kenntnis spezifischer Zeichenressourcen hypertextuell strukturierter Internetseiten voraus, die Meier in einem weiteren Schritt zu differenzieren sucht. Hierbei plädiert er für die Konzepte der «Kohäsion» (auf der Ebene der Gestaltung und des Ausdrucks) und der «Kohärenz» (auf der Ebene des Inhalts), um übergreifende Zusammenhänge und Zugehörigkeiten zwischen einzelnen Elementen von Webseiten fassbar zu machen. Das vorletzte Kapitel beschäftigt sich mit Fragen der Auswahl und Erhebung der Daten. Heuristischen Wert hat hier vor allem die Typologie an Webseiten, die Meier am Gegenstand entwickelt, um unterschiedliche Internetseiten in ihrer kommunikativen Funktionalität voneinander abzugrenzen. Im letzten Teil der Arbeit stellt er Beispielanalysen zum Online-Diskurs der zweiten Ausstellungsversion vor, die angesichts der Tiefe und Breite der vorangegangenen theoretisch-methodologischen Reflexionen allerdings vergleichsweise deskriptiv anmuten.

Wenngleich Stefan Meier ein flüssiger und durchaus anschaulicher Sprachstil zu attestieren ist, wird sich dem Laien das Buch nur schwer erschließen. Zum einen ist das Werk mit mehr als 470 Seiten recht umfangreich und Einleitung sowie Zusammenfassung und Schluss fallen im Vergleich zum gesamten Textkörper knapp aus. Zum anderen richtet es sich als Qualifizierungsarbeit an eine Leserschaft, welche mit semiotischen und diskurstheoretischen Grundlagen und den hiermit verbundenen Begrifflichkeiten bereits vertraut ist. Beachtung verdient die Darstellung vor allem im Anspruch, überindividuelle Einflüsse auf individuelles kommunikatives Handeln sichtbar zu machen, mögliche Bezüge zwischen singulären Kommunikaten herauszuarbeiten sowie sprachliche und ästhetische Ausdrucksweisen nicht isoliert, sondern im Verbund zu betrachten. Zu ihrer Einordnung in den kommunikations- und medienwissenschaftlichen Forschungskontext bleibt indes auch auf die Grenzen der entfalteten diskurstheoretischen Perspektive hinzuweisen: Das Konzept und die Vorgehensweise von Stefan Meier ist vor allem ein Beitrag für die Medien- bzw. Webanalyse. Aus Sicht der Rezeptions- und Aneignungsforschung wäre auch nach den Fähigkeiten, Voraussetzungen und sozialen Kontexten der Rezipient/innen bzw. der aktiven Protagonist/innen zu fragen, die hinter ihren sprachlichen und ästhetischen Aus- 
drücken gleichsam unsichtbar bleiben. Ebenso unberücksichtigt sind freilich medienpädagogische Fragestellungen, wenngleich sie sich nahtlos an die Darstellung anschließen. Diese betreffen beispielsweise die visuelle Kompetenz sowie die Reflexions- und Abstraktionsfähigkeiten, die in der Wahrnehmung und Dekonstruktion übergreifender Diskursmuster in Wort, Bild und Webdesign gefordert sind. Nicht zuletzt mit Blick auf den thematischen Gegenstand der Arbeit und das Wirken rechtsextremer Akteur/innen im Internet verleitet ein solcher Ausblick zu eher pessimistischen Prognosen und es bedarf wohl kaum dem Hinweis, dass (auch) hier Handlungsbedarf besteht.

Wolfgang Reißmann 